\title{
Repair of primary or complicated aortic coarctation in the adult with cardiopulmonary bypass and hypothermic circulatory arrest
}

\author{
Nicholas T. Kouchoukos, MD, James R. Scharff, MD, and Catherine F. Castner, BSN
}

\begin{abstract}
Objective: Patients with primary aortic coarctation or complications of a previous coarctation repair may seek treatment as adults. Management options include open, endovascular, hybrid, and extra-anatomic repairs. We evaluated the results of open direct repair with total cardiopulmonary bypass and hypothermic circulatory arrest.

Methods: Twenty-seven patients with primary coarctation with or without associated aortic abnormalities or with complications of a previous coarctation repair were treated during a 20-year interval. Mean age was 37 years (range, 15-71 years). Thirteen patients had primary coarctation ( 7 with associated aneurysms), 3 patients had aneurysms of the aorta above or below a noncritical narrowing of the aorta at the isthmus ("pseudocoarctation"), and 11 patients had previous repair of a coarctation. The previous repairs were bypass grafting of the coarctation $(n=5)$, interposition grafting $(n=3)$, patch angioplasty $(n=2)$, and primary repair $(n=1)$.
\end{abstract}

Results: Mean durations of cardiopulmonary bypass, hypothermic circulatory arrest and hypothermic fibrillation were 104, 36, and 76 minutes, respectively. There were no in-hospital deaths, and no patients sustained stroke, spinal cord ischemic injury, or renal or respiratory failure. Thirteen patients received no blood products. No patient has had evidence of recurrent coarctation or aneurysm formation.

Conclusions: Cardiopulmonary bypass with hypothermic circulatory arrest is a safe and suitable technique for treatment of primary and recurrent coarctation. It is associated with low operative risk and absence of major complications. (J Thorac Cardiovasc Surg 2015;149:S83-5)

Several options exist for the operative management of coarctation of the aorta and its complications in both adolescent and adult patients. These include open repair with end-to-end anastomosis; interposition, patch, or bypass grafting; balloon angioplasty; endovascular stent grafting; extra-anatomic bypass grafting; and hybrid procedures. Open repair can be performed with simple aortic clamping, partial (left heart) bypass, or total cardiopulmonary bypass (CPB), with or without hypothermia and circulatory arrest. We present here our experience with total CPB and hypothermic circulatory arrest (HCA) for open repair of primary, recurrent, and complex aortic coarctation in adolescents and adults.

\section{MATERIALS AND METHODS \\ Study Population}

During a 20-year interval ending in September 2012, a total of 27 patients underwent repair of aortic coarctation with $\mathrm{CPB}$ and HCA.

\footnotetext{
From the Division of Cardiovascular and Thoracic Surgery, Missouri Baptist Medical Center, St Louis, Mo.

Disclosures: Nicholas T. Kouchoukos reports consulting fees from Kips Bay Medical. All other authors have nothing to disclose with regard to commercial support.

Read at The American Association for Thoracic Surgery Aortic Symposium, New

York, New York, April 24-25, 2014.

Received for publication May 30, 2014; accepted for publication July 24, 2014; available ahead of print Sept 8, 2014.

Address for reprints: Nicholas T. Kouchoukos, MD, Cardiac, Thoracic, and Vascular

Surgery, 3009 N Ballas Rd, Suite 360C, St Louis, MO 63131 (E-mail: NTKouch@ aol.com).

$0022-5223 / \$ 36.00$

Copyright (C) 2015 by The American Association for Thoracic Surgery

http://dx.doi.org/10.1016/j.jtcvs.2014.07.071
}

No other technique of repair was used during this period. The mean age of the patients was 37 years (range, $15-71$ years), and 19 patients $(70 \%)$ were male. Thirteen patients underwent primary repair, and only 5 of these patients had coarctation without associated abnormalities of the aorta or subclavian arteries. The coarctation was located in the region of the aortic isthmus in 12 of these patients. The remaining patient had a "midaortic syndrome," with narrowing of the aorta from the level of the superior mesenteric artery to the aortic bifurcation. Three patients had aneurysmal dilatation of the aorta above or below a narrowing of the aorta at the isthmus without a substantial pressure gradient at this level ("pseudocoarctation"). Eleven patients had undergone previous repair of the coarctation. The initial procedures performed in this group and the resulting complications and indications for reoperation are shown in Table 1. A magnetic resonance angiogram from a patient with an anastomotic pseudoaneurysm of a previously placed bypass graft is shown in Figure 1. The institutional review board of our institution approved this retrospective study and waived the need for individual patient consent.

\section{Operative Technique}

Our technique for repair of aortic coarctation and its complications with $\mathrm{CPB}$ and HCA has been previously reported. ${ }^{1}$ After insertion of a doublelumen endotracheal tube and appropriate monitoring devices, a standard posterolateral thoracotomy incision is made through the fourth intercostal space. Two lines for arterial perfusion are connected to the main arterial line of the pump-oxygenator to permit separate flow to the upper body and lower body as indicated. The left common femoral artery and vein are exposed through an oblique incision in the groin. The vein is cannulated with a long cannula (28F-34F), and the tip is positioned in the superior vena cava under transesophageal echocardiographic guidance. The femoral artery is cannulated with a short $18 \mathrm{~F}, 20 \mathrm{~F}$, or $22 \mathrm{~F}$ cannula. CPB is established and cooling is initiated. If ventricular distention occurs, the left side of the heart is vented through the left inferior pulmonary vein or the apex of the left ventricle. Modifications of this perfusion strategy may be necessary. If there is substantial distention of the left ventricle from aortic regurgitation, as occurred in 1 case, an arterial perfusion 


\section{Abbreviations and Acronyms \\ $\mathrm{CPB}=$ cardiopulmonary bypass \\ $\mathrm{HCA}=$ hypothermic circulatory arrest}

cannula is inserted through the apex of the left ventricle and positioned in the aortic root under echocardiographic guidance. This cannula is attached to the second arterial line from the pump-oxygenator, and approximately $35 \%$ of the total arterial flow is directed through this line. If the coarctation is severe without adequate collateral vessels, the left axillary artery is cannulated and flow to the upper body during cooling is provided with the second arterial line.

During cooling, the distal aortic arch and the area of the coarctation are carefully mobilized. The intercostal and large collateral arteries in the region of the coarctation are not mobilized. The left phrenic, vagus, and recurrent laryngeal nerves are identified and protected. When the nasopharyngeal temperature has reached $15^{\circ} \mathrm{C}$ to $17^{\circ} \mathrm{C}$ and there is electroencephalographic silence, circulatory arrest is established. A clamp is placed on the descending thoracic aorta below the coarctation. Cardioplegic solution is not administered. The aorta is incised, and the orifices of the arch branches are identified. This is important, because in 3 of our cases the right subclavian artery arose as the fourth branch of the aorta. It was preserved in 1 case, reimplanted into the aortic graft in 1 case, and bypassed in the third case. The aorta is transected just distal to the origin of the left subclavian artery, or, if the subclavian artery will require replacement, distal to the origin of the left carotid artery. An appropriately sized impregnated polyester graft is sutured to the aorta with a 4-0 or 5-0 polypropylene suture buttressed with a strip of polytetrafluoroethylene felt. As this suture line is being completed, cold, oxygenated blood is perfused retrogradely through the venous cannula to assist in the evacuation of air from the upper body circulation and from the graft. If a prolonged period of HCA is required, flow to the lower body can be initiated from the femoral artery cannula.

A clamp is then placed on the graft, and the site for anastomosis of the graft to the distal aorta is identified. The aorta is beveled to preserve patent intercostal and collateral arteries, and the graft is sutured to the aorta with a 4-0 or 5-0 polypropylene suture buttressed with a strip of felt. As this suture line is being completed, the clamp on the distal aorta is removed, and perfusion from the femoral artery is initiated. Air is evacuated from the graft with an 18-gauge needle, flow is reestablished, and rewarming is initiated. CPB is discontinued once the bladder temperature has reached $35^{\circ} \mathrm{C}$.

Reconstruction of the left subclavian artery was required in 9 cases. In 2 of these cases, extensive resection of the artery was required because of aneurysmal degeneration, and the anastomosis of a separate graft to the subclavian artery at the level of the thoracic inlet was accomplished during the interval of HCA. In the remaining 7 cases, this anastomosis was performed during the period of rewarming. In all cases, the proximal anastomosis of the subclavian artery graft to the aortic graft was performed during rewarming with a side-biting clamp on the aortic graft.

The patient with severe narrowing of the abdominal aorta required a bypass graft from the proximal descending thoracic aorta to the aorta just proximal to the aortic bifurcation and separate branch grafts from the aortic graft to the right and left renal arteries.

Another patient with recurrent coarctation after placement of an interposition graft also had stenosis of an aberrant right subclavian artery originating proximal to the area of coarctation and hypoplasia of the aortic arch, both of which were not recognized at the time of the original operation. The repair was performed through a median sternotomy incision with HCA. The aortic arch and the area of coarctation were replaced with a
TABLE 1. Previous coarctation repairs

\begin{tabular}{lcl}
\hline \multicolumn{1}{c}{ Initial repair } & No. of patients & Presenting feature \\
\hline Bypass graft & 5 & False aneurysm \\
Interposition graft & 3 & Recurrent stenosis \\
Patch angioplasty & 2 & Patch aneurysm \\
Resection with end-to-end & 1 & Recurrent stenosis \\
$\quad$ anastomosis & & \\
Total & 11 & \\
\hline
\end{tabular}

20-mm graft. The right carotid, left carotid, and left subclavian arteries arose from a common trunk and were attached to the graft with a cuff of aortic tissue. The aberrant right subclavian artery was ligated at its origin. A right subclavian artery to the right carotid artery bypass procedure was performed after discontinuation of CPB.

The cardiopulmonary perfusion data are shown in Table 2. The mean duration of HCA was 36 minutes (range, 25-53 minutes). Thirteen patients received no blood products either during or after the operative procedure. The average transfusion requirements for the remaining 14 patients were 1.2 units of red blood cells, 1.0 units of fresh-frozen plasma, and 0.1 units of platelets.

\section{RESULTS}

There were no in-hospital deaths. The mean stay in the intensive care unit was 3 days (range, 1-8 days). The mean hospital stay was 8 days (range, 4-20 days). No patients required reoperation for bleeding. No patients had development of spinal cord ischemic injury, stroke, renal failure, myocardial failure, left recurrent laryngeal nerve injury, or chylothorax. The mean duration of ventilator support was 0.93 days (median, 1 day), and no patients required tracheostomy. One patient had empyema and a perigraft abscess develop after repair of a patch aneurysm and was treated with topical and intravenous antibiotics. At last follow-up (18 months), there was no evidence for infection.

During the follow-up period, there has been no evidence for recurrent coarctation. No reoperations on the aorta have been required by any of the patients.

\section{DISCUSSION}

Despite the increasing use of endovascular techniques for the management of primary or recurrent aortic coarctation, open surgical repair remains an important therapeutic option. ${ }^{2-4}$ Open repair is of particular value in adolescent or adult patients with recurrent coarctation, aneurysmal degeneration of the adjacent aorta and subclavian arteries, and hypoplasia of the aortic arch. Several methods for open repair of these complex forms of aortic coarctation have been reported. These include direct repair with various techniques, extra-anatomic bypass, and hybrid procedures. ${ }^{1,4-6}$ We have favored direct repair with total $\mathrm{CPB}$ and HCA. We believe that this technique offers distinct advantages relative to other techniques of direct repair. These include the need for only minimal dissection of the tissues surrounding the area of coarctation, which 


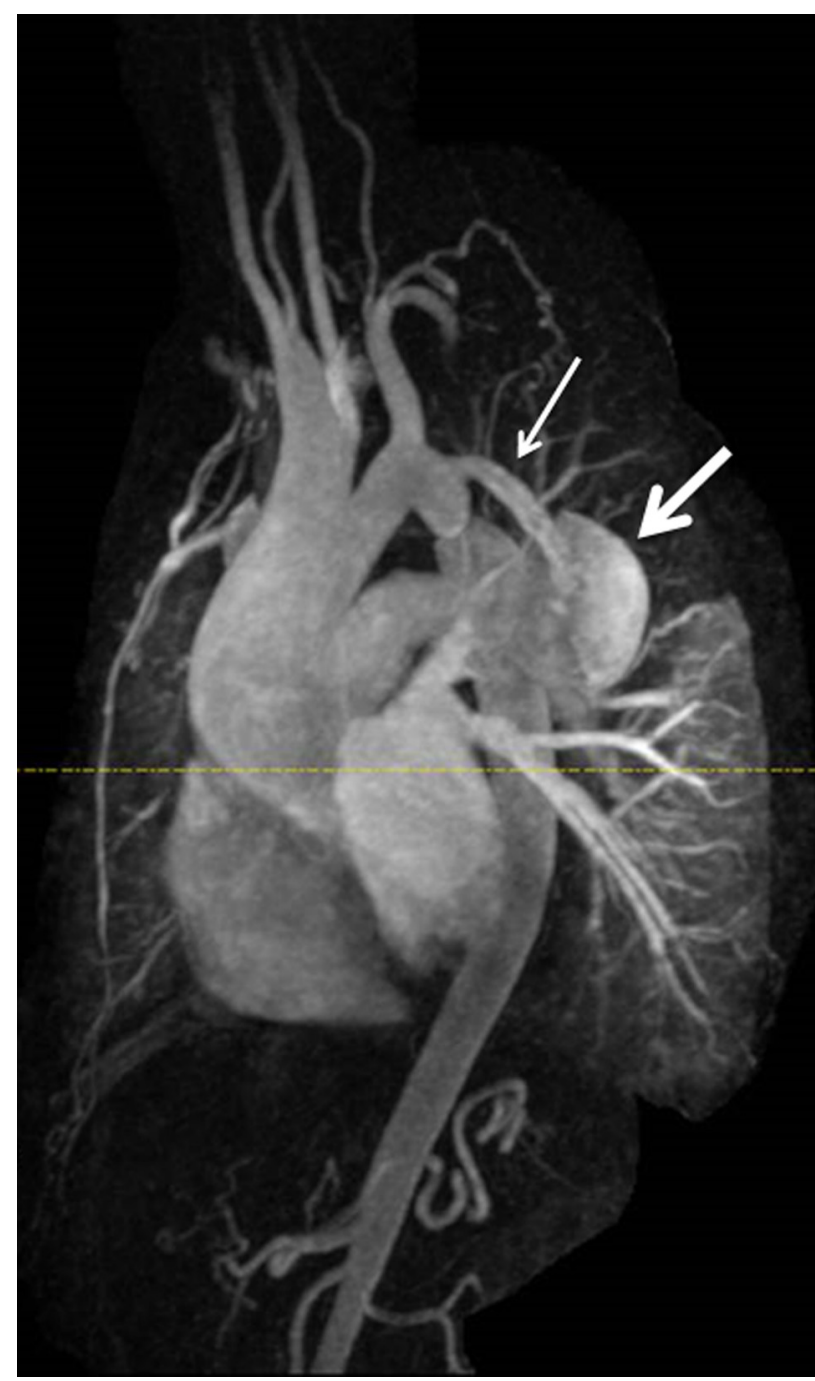

FIGURE 1. Magnetic resonance angiogram from a patient with a pseudoaneurysm (large arrow) at the site of the distal anastomosis of a bypass graft (small arrow) previously placed to treat an aortic coarctation.

greatly reduces the risk of injury to large intercostal and collateral arteries and excessive bleeding; avoidance of clamping the sometimes fragile aorta proximal and distal
TABLE 2. Cardiopulmonary perfusion data

\begin{tabular}{lcc}
\hline & Mean \pm SD & Range \\
\hline Time (min) & & \\
Cardiopulmonary bypass & $104 \pm 36.0$ & $65-230$ \\
Cooling & $33.0 \pm 6.8$ & $24-50$ \\
Circulatory arrest & $35.5 \pm 7.8$ & $25-53$ \\
Hypothermic ventricular fibrillation & $75.5 \pm 15.7$ & $36-112$ \\
Rewarming & $56.2 \pm 17.3$ & $40-130$ \\
Temperature $\left({ }^{\circ} \mathrm{C}\right)$ & & \\
Lowest nasopharyngeal & $16.0 \pm 2.3$ & $12-22$ \\
Lowest bladder or rectal & $19.7 \pm 3.0$ & $15-27$ \\
\hline
\end{tabular}

to the involved segment; clear identification of all relevant anatomy in a bloodless field; the potential to correct hypoplasia of the aortic arch and aneurysms of the aorta and subclavian arteries; and adequate protection of the brain, spinal cord, and myocardium. Our experience to date indicates that direct repair with total CPB and HCA is a safe and suitable technique, particularly for patients with complex or recurrent coarctation, and it remains our technique of choice.

\section{References}

1. Rokkas CK, Murphy SF, Kouchoukos NT. Aortic coarctation in the adult: management of complications and coexisting arterial abnormalities with hypothermic cardiopulmonary bypass and circulatory arrest. J Thorac Cardiovasc Surg. 2002; 124:155-61.

2. Carr JA. The results of catheter-based therapy compared with surgical repair of adult aortic coarctation. J Am Coll Cardiol. 2006;47:1101-7.

3. Rodés-Cabau J, Miró J, Dancea A, Ibrahim R, Piette E, Lapierre C, et al. Comparison of surgical and transcatheter treatment for native coarctation of the aorta in patients $\geq 1$ year old. The Quebec Native Coarctation of the Aorta Study. Am Heart J. 2007;154:186-92.

4. Brown ML, Burkhart HM, Connolly HM, Dearani JA, Hagler KJ, Schaff HV. Late outcomes of reintervention on the descending aorta after repair of aortic coarctation. Circulation. 2010;122(11 Suppl):S81-4.

5. McKellar SH, Schaff HV, Dearani JA, Daly RC, Mullany CJ, Orszulak TA, et al. Intermediate-term results of ascending-descending posterior pericardial bypass of complex aortic coarctation. J Thorac Cardiovasc Surg. 2007;133: 1504-9.

6. Roselli EE, Qureshi A, Idrees J, Lima B, Greenberg RK, Svensson LG, et al. Open, hybrid, and endovascular treatment for aortic coarctation and postrepair aneurysm in adolescents and adults. Ann Thorac Surg. 2012;94:751-6; discussion $757-8$. 